\title{
Etiologia e tratamento da estomatite aftosa recorrente - revisão de literatura
}

\author{
Etiology and treatment of recurrent aphthous \\ stomatitis - literature review
}

\author{
Gilliene B. F. Costa ${ }^{1}$, Jurema F. L. Castro²
}

\begin{abstract}
RESUMO
Objetivos: Descrever as características e os principais achados relatados na literatura quanto à provável etiologia e tratamentos das lesões causadas pela Estomatite Aftosa Recorrente (EAR). Métodos: Realizou-se a revisão da literatura nas bases de dados PubMed, SciELO e LILACS, no período de tempo do ano de 2005 a 2012. Utilizaram-se, isoladamente e em combinação, os descritores a seguir: Stomatitis, Aphthous; Oral Ulcer; Risk Factors e Therapeutics. Resultados: A patogênese da estomatite aftosa recorrente ainda continua indefinida, havendo confirmação científica quanto à sua relação com fatores imunológicos e mutações genéticas. Os procedimentos realizados e recursos utilizados para o tratamento das lesões são paliativos, no intuito de aliviar a dor, não existindo uma terapêutica de cura. Conclusão: Por ser a etiologia da EAR ainda incerta, o tratamento atual das lesões é bastante variado, e baseia-se no alívio dos sintomas da doença, variando, desde o uso de produtos naturais, como a própolis, passando por anti-inflamatórios, até à aplicação do laser de baixa potência.
\end{abstract}

Palavras-chave: Estomatite Aftosa. Úlceras Orais. Fatores de Risco. Terapêutica.

\section{Introdução}

A Estomatite Aftosa Recorrente (EAR) consiste em uma condição comum da cavidade oral ${ }^{1,2}$, a qual é caracterizada pela presença de lesões em mucosa oral, que podem ocorrer de forma simples ou múltipla, ${ }^{3}$ e são classificadas em três tipos: menor, maior e herpetiforme. Além do tamanho, essas lesões também diferem em relação aos seus tempos de duração e à formação de cicatrizes. ${ }^{4}$
Essa afecção possui diversas causas, no entanto, nenhuma estabelecida de forma precisa. Por a etiologia ainda ser desconhecida, não há um tratamento estabelecido para a cura das lesões até o momento. Todas as formas de tratamento são voltadas para o alívio dos sintomas e cicatrização das úlceras. ${ }^{5}$ Os medicamentos usados reduzem a dor e a frequiência de aparecimento das lesões. Esses tratamentos podem ser administrados de forma tópica ${ }^{1,5-8}$ ou sistêmica, ${ }^{2,9-13}$ através do uso de medicamentos alopáticos,
1. Aluna do Curso de Mestrado em Odontologia, Universidade Federal de Pernambuco, Pernambuco, Brasil.

2. Doutora em Estomatologia, Professora Associada da Universidade Federal de Pernambuco, Pernambuco, Brasil.
Correspondência: Gilliene Batista Ferreira da Costa Avenida Conde da Boa Vista, 1482. Apt. 111. Boa Vista. Recife. Pernambuco. Brasil. CEP: 50.060-001.

Fone: (81) 8756-4627 Email: gillienecosta@ hotmail.com

Artigo recebido em 27/08/2012 Aprovado para publicação em 18/12/2012 
homeopáticos ou produtos naturais ${ }^{14,-17}$, além das formas alternativas de tratamento como a aplicação do laser de baixa potência.

A terapêutica para essa lesão é significativamente importante, já que o paciente é acometido por dor e alteração das funções orais, tais como comer e deglutir. ${ }^{4}$ Além disso, quando está envolvido o fator de história familiar pregressa de acometimento por essa doença, há maior severidade das lesões, com seus aparecimentos em idade cada vez mais precoce. ${ }^{5}$

Pela relevância do tema, este artigo tem como objetivo descrever as características, e os principais achados relatados na literatura quanto à provável etiologia e tratamentos das lesões causadas pela Estomatite Aftosa Recorrente.

\section{Métodos}

Este artigo de revisão é produto de investigação exploratória e bibliográfica realizada nas bibliotecas eletrônicas PubMed, SciELO e LILACS, através da consulta a artigos científicos publicados em periódicos nos últimos sete anos (2005 - 2012), dando ênfase ao embasamento teórico-conceitual do assunto abordado. Na busca, foram utilizados, isoladamente e em combinação, os seguintes descritores: Stomatitis, Aphthous; Oral Ulcer; Risk Factors e Therapeutics. Do material pesquisado encontrado, foram selecionadas as referências que contribuíram para o cumprimento do objetivo deste trabalho, ou seja, descrever as características e os principais achados relatados na literatura quanto à provável etiologia e tratamentos das lesões causadas pela EAR. A investigação foi encerrada quando surgiram sinais de saturação teórica do tema da pesquisa.

\section{Características clínicas e classificação}

A Estomatite Aftosa Recorrente ou Úlcera Aftosa Recorrente é uma das afecções mais comuns e intrigantes da cavidade oral. ${ }^{6,18,19,20}$ Consiste na perda súbita do tecido normal da mucosa oral, sendo lesões recorrentes, dolorosas, até mesmo incapacitantes ${ }^{21}$ redondas ou ovaladas, com halo eritematoso. Começam a aparecer na infância, porém possuem maior frequiência em adolescentes e adultos jovens. ${ }^{22}$

Para que o paciente se enquadre como portador da EAR, ele deve apresentar aftas orais em períodos mínimos quinzenais (ou mensais), por mais de um ano de duração, sem que haja sinais de doença sistêmica associada (como por exemplo doença de Behcet ou doença celíaca). ${ }^{23}$
Os sintomas ocasionados pela EAR levam o paciente a alterar sua rotina diária, já que incluem dor e debilidade. ${ }^{9}$ Também afetam a função oral normal, prejudicando a fala, a mastigação e a deglutição, $, 21,24$ levando à deficiência nutricional e à falta de qualidade de vida. ${ }^{4,25,26}$

Essas lesões são classificadas em três tipos: menor, maior e herpetiforme. Geralmente, a lesão de Estomatite Aftosa Recorrente Menor, a mais comum dentre os três tipos, tem dimensão menor que $10 \mathrm{~mm}$ e duração entre 10 e 14 dias. Essas aftas, presentes comumente na mucosa não-ceratinizada, de forma única ou múltipla, dolorosas, arredondadas, com bordas regulares avermelhados e uma base de coloração cinza-amarelada. ${ }^{27}$ As lesões do tipo maior são incomuns, no entanto, são as mais severas, com duração de semanas ou meses, resultando comumente em cicatrizes. ${ }^{4}$ São ovais e podem até mesmo exceder um $\mathrm{cm}$ de diâmetro, acometendo principalmente lábios e palato mole. A forma herpetiforme é a menos comum delas. É caracterizada pela presença de aftas múltiplas e pequenas $(1-2 \mathrm{~mm})$ que coalescem, formando úlceras maiores. ${ }^{28,29}$ (Figuras 1-3).

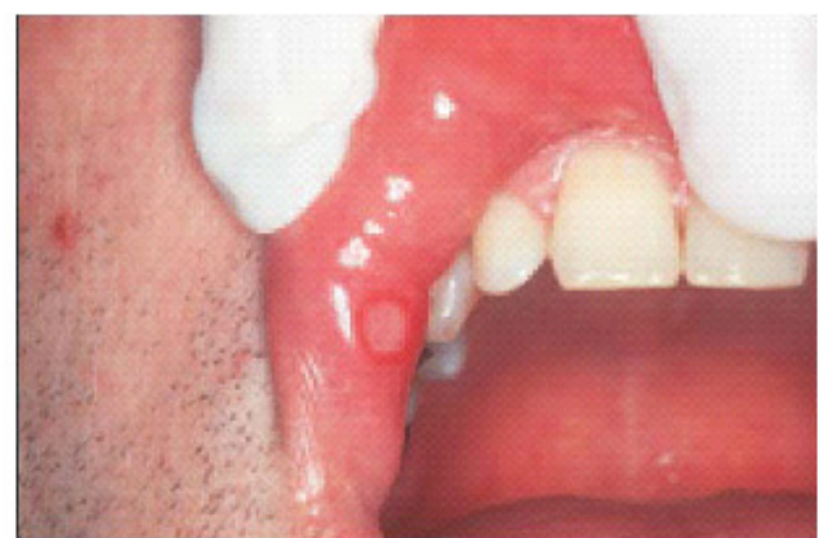

Figura. 1. Estomatite aftosa menor ${ }^{29}$

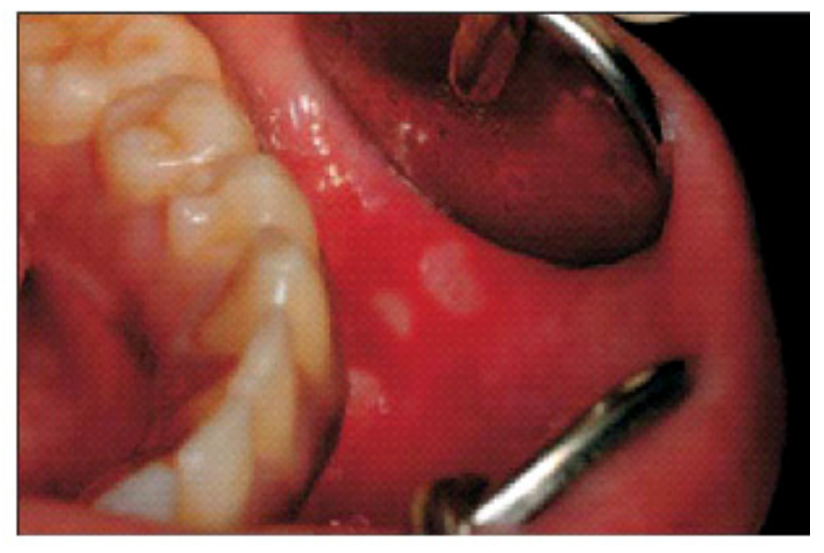

Figura. 2. Estomatite aftosa maior ${ }^{29}$ 


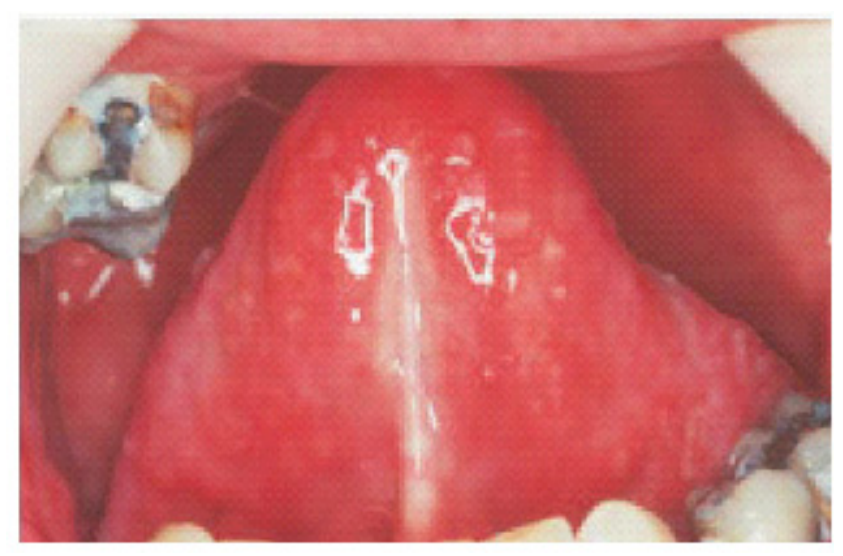

Figura. 3. Estomatite aftosa herpetiforme ${ }^{29}$

\section{Etiologia}

A etiologia da EAR, apesar de ser alvo de inúmeras pesquisas, ainda é desconhecida5 e classificada como multifatorial, ${ }^{18,27}$ sendo esses fatores causais de origem local ou sistêmica. ${ }^{20,22}$ No entanto, quando se afirma que a causa é desconhecida, não se exclui que há sustentações científicas bem estabelecidas quanto à etiologia. Sabe-se perfeitamente que há correlação entre a EAR e a ocorrência de doença auto-imune, deficiência nutricional e alergia a alimentos. ${ }^{3,30,31}$ Além de sua associação com a presença de hiperacidez bucal ${ }^{28}$, história familiar pregressa da doença $^{32,33}$ e estresse emocional ${ }^{34}$ ou fisiológico. ${ }^{35}$

Dentre esses fatores, a inadequação de defesa do organismo, expressado através das doenças autoimunes, tem um papel de destaque na patogênese da EAR,,$^{33}$ a exemplo do que ocorre quando do acometimento por doença auto-imune da tireóide, a qual tem sido relacionada com casos de pacientes com EAR. Essa relação causal pode ser observada através da expressão da citocina Th1 com a etiopatogenia da doença, já que pacientes com doença auto-imune da tireóide apresentam maior relação com aparecimento de úlceras orais. ${ }^{30}$ Além dessa citocina, o fator alfa de necrose tumoral pode estar vinculado ao aparecimento de novas úlceras na cavidade oral, já que ele pode estimular a expressão do complexo principal de histocompatibilidade, desencadeando uma resposta citotóxica e ulceração da mucosa..$^{20,30}$

Ainda confirmando a relação causal da EAR com a imunidade dos pacientes, está o acometimento de grande parte dos pacientes com Aids por úlceras orais, o que pode levar a uma associação entre a imunossupressão e um aumento na severidade das lesões orais. ${ }^{4}$ Dessa forma, na prática clínica, diante da ocor- rência de úlceras orais persistentes, deve-se ter como prática a solicitação de exames laboratoriais complementares que demonstrem o estado imunológico do paciente, a fim de guiar o profissional da saúde no diagnóstico e estabelecimento do procedimento terapêutico adequado.

No entanto, na prática clínica diária, a maioria dessas lesões não está relacionada a doenças ou síndromes, ${ }^{28}$ apesar de algumas síndromes trazerem a EAR como um fator constante. Essa associação com síndromes conduz estudos em busca da correlação entre a etiologia da EAR e fatores genéticos, ${ }^{36}$ a exemplo do que possivelmente ocorre nos casos em que há uma forte associação entre a ocorrência de úlceras orais e história familiar da doença por gerações. ${ }^{24}$

Essa questão, no que diz respeito ao aspecto familiar e genético da EAR, pode ser evidenciada através de mutações no MEFV, gene relacionado com a febre mediterrânea familiar, principalmente mutação no gene M694V, o qual atua como um fator atenuante da gravidade em pacientes com EAR portadores da síndrome de PFAPA (febre periódica, estomatite aftosa, faringite, estomatite e adenopatia). ${ }^{36}$

No entanto, o envolvimento genético na etiologia da EAR não se faz presente apenas nos casos em que há histórico familiar da ocorrência de lesões orais, mas também em pacientes diversos. Essa correlação é confirmada também por o polimorfismo do IL-1 beta estar associado ao aumento do risco de desenvolvimento da EAR. ${ }^{37}$ Além disso, as moléculas que estão diretamente implicadas no controle genético da resposta imune, como o HLA-A33 e o HLA-B35, mais especificamente na população brasileira, estão fortemente associadas com a etiologia e a ocorrência da estomatite aftosa recorrente do tipo menor. ${ }^{38}$

Essas revelações do envolvimento genético nos pacientes acometidos por lesões decorrentes da EAR, com o estabelecimento dos genes e moléculas envolvidas, constitui um grande passo na descoberta da etiologia da EAR, já que vai possibilitar o estabelecimento de formas de tratamento que ajam diretamente na causa da doença, e não apenas no alívio dos sintomas, como ainda acontece.

\section{Tratamento}

Devido à falta de precisão no estabelecimento da causa da EAR, a terapêutica não é específica, sendo utilizados, de forma paliativa, anti-inflamatórios, antibióticos e anestésicos, ${ }^{1,5-8,39}$ além de medicamentos com propriedades naturais e homeopáticos, ${ }^{40,41,42}$ para 
alívio da dor, ${ }^{9,43}$ redução da severidade, duração e frequência de aparecimento das úlceras ${ }^{3}$ e o restabelecimento das funções orais normais. ${ }^{44}$

A utilização desses diferentes recursos terapêuticos se dá tanto de forma sistêmica, quanto de forma tópica. No entanto, por a forma tópica de tratamento ser mais eficiente e segura para o paciente, com a ocorrência de menor quantidade de efeitos colaterais, essa consiste na primeira opção de tratamento para as aftas recorrentes. . $^{1,5-8,39}$

Dentre os recursos tópicos utilizados com eficiência para redução e controle da dor na EAR estão as soluções orais para bochecho. Seja contendo o anestésico benzocaína ${ }^{8}$ em sua fórmula, os antibióticos minociclina $0,2 \%$ e tetraciclina $0,25 \%,{ }^{1,27}$ ou o ácido lático 5\%, a administração tópica através de bochecho reduz os sinais e sintomas da doença através do aumento da secreção espontânea de fator de crescimento endotelial pelos queratinócitos. ${ }^{45}$

Outro agente terapêutico utilizado no processo de cura de pequenas aftas, assim como no tratamento de colites e úlceras gástricas, consiste na quercetina, a qual foi tida como uma droga segura, bem tolerada e altamente eficaz. ${ }^{19}$ Do mesmo modo, o ácido hialurônico $0,2 \%$ reduz imediatamente a dor após aplicação a aplicação tópica. ${ }^{46}$

Além desses recursos halopáticos, os produtos naturais vêm ganhando espaço no tratamento tópico das úlceras orais. Os extratos aquosos da casca do Mangue-vermelho (Rizophora mangle) ${ }^{14} \mathrm{e}$ do extrato de alcaçuz, ${ }^{15}$ além da própolis, mostram-se eficazes na redução dos sinais e sintomas da EAR. ${ }^{16,17}$

No entanto, apesar dessa inúmera quantidade de opções de tratamento tópico, os antiinflamatórios são os mais utilizados, sendo o amlexanox $5 \%$ sob a forma de película, preferido pelos pacientes por ser mais confortável do que sob a forma de tablete. ${ }^{5}$

Apesar de ser seguro ao paciente, quando o tratamento tópico não é eficiente ${ }^{47}$ ou a severidade da doença é maior, com manifestações severas, instituise o tratamento sistêmico, ${ }^{2,9-13,48}$ mesmo diante da ocorrência de efeitos colaterais diversos, ${ }^{5,49}$ como: dor abdominal, náusea, fadiga, diarreia, vertigem, letargia, cefaleia, hemólise, icterícia e decréscimo no nível de hemoglobina. ${ }^{9}$

Os medicamentos sistêmicos mais utilizados no tratamento da EAR com manifestações severas ${ }^{2,9-13}$ ainda são a talidomida, dapsone e colchicina, apesar de causarem a maioria desses efeitos adversos no paciente, já que apresentarem propriedades anti-infla- matórias e imunológicas capazes de atuar na redução e na prevenção do desenvolvimento das lesões. ${ }^{9-13}$

No entanto, outras formas terapêuticas, como a clofazimina e a prednisolona, já apresentam melhores resultados no tratamento da EAR, quando comparadas à colchicina e à talidomida, por não causarem efeitos gastrointestinais adversos, ${ }^{2,13}$ nem teratogenicidade ou neuropatia. ${ }^{3}$ Já a pentoxifilina, droga de propriedades vasodilatadoras, apresenta eficácia limitada no tratamento das lesões, além de proporcionar efeitos colaterais ao paciente. ${ }^{50}$

Por esses efeitos adversos muitas vezes limitar e interromper o tratamento, os anti-inflamatórios esteroides, quando administrados por um curto período de tempo, são opções viáveis para o tratamento das lesões, já que não ocasionam efeito colateral significativo, aliviam os sintomas e diminuem o desconforto dos pacientes, a exemplo da prednisona. ${ }^{9,48}$ Eles agem reduzindo a atuação dos granulócitos e dos leucócitos (células envolvidas no processo inflamatório), estabilizam a membrana dos lisossomos, mantêm a integridade da membrana celular, inibem a fagocitose e a proliferação dos fibroblastos. ${ }^{21}$

Do mesmo modo, os produtos de origem natural, quando administrados sistemicamente, também são viáveis por não causarem efeitos adversos, a exemplo da própolis, o qual proporciona aos pacientes uma melhora em suas qualidades de vida, ${ }^{49}$ além de ser um recurso de baixo custo. ${ }^{16,17}$

Como visto, existe uma variedade de tratamento para as lesões da EAR. No entanto, por as lesões serem de causa multifatorial, estabelecer primeiramente suas etiologias consiste no procedimento ideal antes de se iniciar qualquer terapêutica, não visando apenas o alívio dos sintomas, mas a resolução da doença como um todo. ${ }^{22}$

Essa afirmação é exemplificada quando se diagnostica um paciente portador de EAR com deficiência nutricional, já que o mesmo pode ser tratado através da administração de vitamina B12 ou vitamina C. Essa consiste em uma resolução simples, barata e de pouco risco aos pacientes com EAR, reduzindo o número de úlceras orais e da dor. ${ }^{31,52} \mathrm{~A}$ vitamina C exerce um papel importante no processo inflamatório. Essa ação é explicada pela sua atuação na apoptose dos neutrófilos, os quais mediam a inflamação. ${ }^{52}$

Diante de todas essas formas de tratamento das lesões da EAR, atualmente, o laser de baixa potência mostra-se como uma das melhores opções para o tratamento das lesões da EAR, já que apresenta inúme- 
ras vantagens ao paciente, atuando ao mesmo tempo de forma anestésica, analgésica e anti-inflamatória. ${ }^{53}$

Quando comparada a ação do laser Nd:YAG, à ação dos medicamentos na terapêutica da EAR, o laser apresenta menos complicações funcionais, além de que, obtém maior aceitação pelos pacientes e possibilita menor tempo de tratamento. ${ }^{54}$ Do mesmo modo age o laser de $\mathrm{CO}_{2}$, com redução imediata da dor causada pelas úlceras orais e ausência de efeitos colaterais. ${ }^{55}$

\section{Conclusões}

A estomatite aftosa recorrente consiste em uma das mais intrigantes condições que acometem os teci- dos da cavidade oral. É caracterizada pela presença de lesões dolorosas, que aparecem em períodos mínimos quinzenais (ou mensais), e persistem por mais de um ano. Apesar de sua etiologia ser incerta, há comprovação científica de sua associação com fatores imunológicos e genéticos. O tratamento atual das lesões é bastante variado, já que não existe um fator causal bem determinado, e baseia-se no alívio dos sintomas da doença, variando, desde o uso de produtos naturais, como a própolis, passando por anti-inflamatórios, até à aplicação do laser de baixa potência. A efetividade completa do tratamento das lesões orais causadas pela EAR só será possível quando determinada com precisão a causa dessa doença.

\begin{abstract}
Objectives: To describe the characteristics and key findings reported in the literature about the possible etiology and treatment of lesions caused by Recurrent Aphthous Stomatitis (RAS). Methods: It was conducted a literature review in the databases PubMed, SciELO and LILACS, from 2005 to 2012. Were used, singly or in combination, the following descriptors: Stomatitis, Aphthous; Oral Ulcer; Risk Factors and Therapeutics. Results: The pathogenesis of recurrent aphthous stomatitis still remains unclear, however, there is strong evidence regarding its relationship with immunological factors and genetic mutations. The procedures performed and resources used for the treatment of lesions are palliative in order to relieve pain, there is not a therapeutic healing. Conclusion: As the uncertain etiology of RAS, the current treatment of lesions is very wide, and relies on relieving symptoms of the disease, ranging from the use of natural products, such as propolis, to anti-inflammatories, until the implementation of low-power laser.
\end{abstract}

Keywords: Stomatitis, Aphthous. Oral Ulcer. Risk Factors. Therapeutics.

\section{Referências}

1. Gorsky M, Epstein J, Rabenstein S, Elishoov H, Yarom N. Topical minocycline and tetracycline rinses in treatment of recurrent aphthous stomatitis: a randomized cross-over study. Dermatol Online J. 2007;13:1.

2. Pakfetrat A, Mansourian A, Momen-Heravi F, Delavarian Z, Momen-Beitollahi J, Khalilzadeh $\mathrm{O}$, et al. Comparison of colchicine versus prednisolone in recurrent aphthous stomatitis: A double-blind randomized clinical trial. Clin Invest Med. 2010; 33:E189-95

3. Wardhana EAD. Recurrent aphthous stomatitis caused by food allergy. Acta Med Indones. 2010;42:236-40.

4. Miziara ID, Araujo Filho BC, Weber R. AIDS and recurrent aphtous stomatitis. Rev Bras Otorrinolaringol. 2005;71:517-20.

5. Meng W, Dong Y, Liu J, Wang Z, Zhong X, Chen R, et al. A clinical evaluation of amlexanox oral adhesive pellicles in the treatment of recurrent aphthous stomatitis and comparison with amlexanox oral tablets: a randomized, placebo controlled, blinded, multicenter clinical trial. Trials. 2009;10:30.

6. Al-Na'mah ZM, Carson R, Thanoon IA. Dexamucobase: a novel treatment for oral aphthous ulceration. Quintessence Int.2009;40:399-404.
7. Rodriguez M, Rubio JA, Sanchez R. Effectiveness of two oral pastes for the treatment of recurrent aphthous stomatitis. Oral Dis. 2007;13:490-4.

8. Shemer A, Amichai B, Trau H, Nathansohn N, Mizrahi B Domb AJ. Efficacy of a mucoadhesive patch compared with an oral solution for treatment of aphthous stomatitis. Drugs R D. 2008;9:29-35.

9. Mimura MAM, Hirota SK, Sugaya NN, Sanches Jr JA, Migliari DA. Systemic treatment in severe cases of recurrent aphthous stomatitis: an open trial. Clinics. 2009; 64:193-8.

10. Shetty K. Thalidomide in the management of recurrent aphthous ulcerations in patients who are HIV-positive: a review and case reports. Spec Care Dentist. 2005; 25:236-41.

11. Hello M, Barbarot S, Bastuji-Garin S, Revuz J, Chosidow O. Use of thalidomide for severe recurrent aphthous stomatitis: a multicenter cohort analysis. Medicine (Baltimore). 2010;89:176-82.

12. Sharquie KE, Najim RA, Al-Hayani RK, Al-Nuaimy AA, Maroof DM. The therapeutic and prophylactic role of oral zinc sulfate in management of recurrent aphthous stomatitis (ras) in comparison with dapsone. Saudi Med J. 2008;29:734-8.

13. Abreu MA, Hirata CH, Pimentel DR, Weckx LL. Treatment of recurrent aphthous stomatitis with clofazimine. Oral Surg Oral Med. Oral Pathol. Oral Radiol. Endod. 2009;108:714-21. 
14. Armas E, Sarracent Y, Marrero E, Fernandez O, BranfordWhite C. Efficacy of Rhizophora mangle aqueous bark extract (RMABE) in the treatment of aphthous ulcers: a pilot study. Curr Med Res Opin. 2005; 21:1711-5.

15. Moghadamnia AA, Motallebnejad M, Khanian M. The efficacy of the bioadhesive patches containing licorice extract in the management of recurrent aphthous stomatitis. Phytother Res. 2009; 23:246-50.

16. Lofuto MA, Lemos Júnior CA, Shimizu MT, Cabral R, Birman G. Clinical evaluation of the topical use of propolis in recurrent minor aphthous ulceration. Ciênc Odontol Bras. 2005;8:6-9.

17. Susana BL, Xiomara MCM. Efectividad del uso del propóleo en el tratamiento de la estomatitis aftosa. Rev Cuba Estomatol. 2007; 44(3).

18. Jiang XW, Hu J, Mian FI. A new therapeutic candidate for oral aphthous ulcer: Allicin. Med Hypotheses. 2008;71:897-9.

19. Hamdy AA, Ibrahem MA. Management of aphthous ulceration with topical quercetin: a randomized clinical trial. J Contemp Dent Pract. 2010 Jul 1;11(4):E009-16.

20. Valle AE, Martinez-Conde-Llamosas R, López-Vicente J, Uribarri-Etxebarria A, Aguirre-Urizar J. Salivary levels of Tumour Necrosis Factor-alpha in patients with recurrent aphthous stomatitis. Med Oral Patol Oral Cir Bucal. 2011;16:e33-6.

21. Quijano D, Rodríguez M. Corticoides tópicos en la estomatitis aftosa recurrente. Revisión sistemática. Acta Otorrinolaringol Esp. 2008; 59:298-307.

22. Toche P, Salinas J, Guzmán MA, Afani A, Jadue N. Úlceras orales recurrentes: características clínicas y diagnóstico diferencial. Rev Chil Infectol. 2007;24: 215-9.

23. Scully C, Gorsky M, Lozada-Nur F. The diagnosis and management of recurrent aphthous stomatitis: a consensus approach. J Am Dent Assoc.2003;134:200-7.

24. Safadi RA. Prevalence of recurrent aphthous ulceration in Jordanian dental patients. BMC Oral Health. 2009; 9:31.

25. Weckx LLM, Hirata CHW, Abreu MAMM, Fillizolla VC, Silva OMP. Levamisol não previne lesões de estomatite aftosa recorrente: um ensaio clínico randomizado, duplo-cego e controlado por placebo. Rev Assoc Med Bras. 2009; 55:1328.

26. Krisdapong S, Sheiham A, Tsakos G. Impacts of recurrent aphthous stomatitis on quality of life of 12 - and 15-year-old Thai children. Qual Life Res. 2012; 21:71-6.

27. Crespo MR, Martínez AB. Aftas de la mucosa oral. Av Odontoestomatol 2011;27:63-74.

28. Miziara ID, Costa KC, Mahmoud A, Weber R, Wilhelmsen NSW, Imamura R. Laryngeal manifestations in atypical recurrent aphthous stomatitis. Braz J Otorhinolaryngol. 2009;75:6604.

29. Scully C, Felix DH. Oral medicine-update for the dental practitioner aphthous and other common ulcers. Br Dent $\mathrm{J}$. 2005;5:259-64.

30. Ozdemir IY, Calka O, Karadag AS, Akdeniz N, Ozturk M. Thyroid autoimmunity associated with recurrent aphthous stomatitis. J Eur Acad Dermatol Venereol. 2012;26:226-30.

31. Gulcan E, Toker S, Hatipoglu H, Gulcan A, Toker A. Cyanocobalamin may be beneficial in the treatment of recurrent aphthous ulcers even when vitamin B12 levels are normal. Am J Med Sci. 2008;336:379-82.

32. Koybasi S, Parlak AH, Serin E, Yilmaz F, Serin D. Recurrent aphthous stomatitis: investigation of possible etiologic factors. Am J Otolaryngol. 2006;27:229-32.
33. Arikan S, Durusoy C, Akalin N, Haberal A, Seckin D. Oxidant/ antioxidant status in recurrent aphthous stomatitis. Oral Dis. 2009;15:512-5.

34. Albanidou-Farmaki E, Poulopoulos AK, Epivatianos A, Farmakis $\mathrm{K}$, Karamouzis M, Antoniades D. Increased anxiety level and high salivary and serum cortisol concentrations in patients with recurrent aphthous stomatitis. Tohoku J Exp Med. 2008; 214:291-6.

35. Gallo CB, Mimura MAM, Sugaya NN. Psychological stress and recurrent aphthous stomatitis. Clinics. 2009;64:645-8.

36. Berkun Y, Levy R, Hurwitz A, Meir-Harel M, Lidar M, Livneh A, et al. The familial Mediterranean fever gene as a modifier of periodic fever, aphthous stomatitis, pharyngitis, and adenopathy syndrome. Semin Arthritis Rheum.2011; 40: 467-72

37. Guimarães AL, Correia-Silva Jde F, Sá AR, Victória JM, Diniz MG, Costa Fde O, et al. Investigation of functional gene polymorphisms IL-1beta, IL-6, IL-10 and TNF-alpha in individuals with recurrent aphthous stomatitis. Arch Oral Biol. 2007;52:268-72.

38. Wilhelmsen NS, Weber R, Monteiro F, Kalil J, Miziara ID. Correlation between histocompatibility antigens and recurrent aphthous stomatitis in the Brazilian population. Braz $\mathrm{J}$ Otorhinolaryngol.;75:426-31.

39. Gorsky M, Epstein J, Raviv A, Yaniv R, Truelove E. Topical minocycline for managing symptoms of recurrent aphthous stomatitis. Spec Care Dentist. 2008;28:27-31.

40. Bratel J, Hakeberg M, Jontell M. The effect of LongoVital on recurrent aphthous stomatitis in a controlled clinical trial. Oral Health Prev Dent. 2005;3:3-8.

41. Mousavi F, Mojaver YN, Asadzadeh M, Mirzazadeh M. Homeopathic treatment of minor aphthous ulcer: a randomized, placebo-controlled clinical trial. Homeopathy. 2009;98:13741.

42. Pourahmad M, Rahiminejad M, Fadaei S, Kashafi H. Effects of camel thorn distillate on recurrent oral aphthous lesions. J Dtsch Dermatol Ges. 2010;8:348-52.

43. Porter SR, Hegarty A, Kaliakatsou F, Hodgson TA, Scully C. Recurrent aphthous stomatitis. Clin Dermatol. 2000; 18: 56978.

44. Hoseinpour H, Peel SA, Rakhshandeh H, Forouzanfar A, Taheri $\mathrm{M}$, Rajabi O, et al. Evaluation of Rosa damascena mouthwash in the treatment of recurrent aphthous stomatitis: a randomized, double-blinded, placebo-controlled clinical trial. Quintessence Int.2011;42:483-91.

45. Sharquie KE, Al-Tammimy SM, Al-Mashhadani S, Hayani RK, Al-Nuaimy AA. Lactic acid 5 percent mouthwash is an effective mode of therapy in treatment of recurrent aphthous ulcerations. Dermatol Online J. 2006;12:2.

46. Nolan A, Baillie C, Badminton J, Rudralingham M, Seymour RA. The efficacy of topical hyaluronic acid in the management of recurrent aphthous ulceration. J Oral Pathol Med. 2006;35:461-5.

47. Femiano F, Lanza A, Buonaiuto C, Gombos F, Nunziata M, Piccolo S, et al. Guidelines for diagnosis and management of aphthous stomatitis. Pediatr Infect Dis J. 2007; 26:728-32.

48. Femiano F, Buonaiuto C, Gombos F, Lanza A, Cirillo N. Pilot study on recurrent aphthous stomatitis (RAS): a randomized placebo-controlled trial for the comparative therapeutic effects of systemic prednisone and systemic montelukast in subjects unresponsive to topical therapy. Oral Surg. Oral Med. Oral Pathol. Oral Radiol. Endod. 2010;109:402-7.

49. Samet N, Laurent C, Susarla SM, Samet-Rubinsteen N. The effect of bee propolis on recurrent aphthous stomatitis: a pilot study. Clin Oral Investig. 2007;11:143-7. 
50. Thornhill MH, Baccaglini L, Theaker E, Pemberton MN. A randomized, double-blind, placebo-controlled trial of pentoxifylline for the treatment of recurrent aphthous stomatitis. Arch Dermatol. 2007;143:463-70.

51. Volkov I, Rudoy I, Freud T, Sardal G, Naimer S, Peleg R. Effectiveness of Vitamin B12 in Treating Recurrent Aphthous Stomatitis: A Randomized, Double-Blind, Placebo-Controlled Trial. JABFM. 2009;22:9-16.

52. Yasui K, Kurata T, Yashiro M, Tsuge M, Ohtsuki S, Morishima T. The effect of ascorbate on minor recurrent aphthous stomatitis. Acta Paediatr. 2010;99:442-5.
53. Bispo LB. A nova tecnologia do laser terapêutico no controle da dor. Rev Bras Odontol. 2009;66:107-11.

54. Tezel A, Kara C, Balkaya V, Orbak R. An evaluation of different treatments for recurrent aphthous stomatitis and patient perceptions: Nd:YAG laser versus medication. Photomed Laser Surg. 2009;27:101-6.

55. Zand N, Ataie-Fashtami L, Djavid GE, Fateh M, Alinaghizadeh MR, Fatemi SM, et al. Relieving pain in minor aphthous stomatitis by a single session of non-thermal carbon dioxide laser irradiation. Lasers Med Sci; 24: 515-20. 